\title{
Development of a Real-Time General Medicine 30-Day Readmissions Notification System
}

\author{
J onathan Bae ${ }^{1}$, Thomas Owens ${ }^{1}$, J effrey M. Ferranti ${ }^{2,}{ }^{3}$, William Gilbert ${ }^{3}$, I lona Stashko ${ }^{3}$, \\ Elizabeth Willis ${ }^{3}$, Tanya Barros ${ }^{3}$, Monica M. Horvath ${ }^{3}$ \\ 1. Division of Hospital Medicine, Department of Medicine, Duke University School of Medicine, Durham, NC, United States. \\ 2. Department of Pediatrics, Duke University School of Medicine, Durham, NC, United States. 3. Duke Health Technology \\ Solutions, Duke University Health System, Durham, NC, United States
}

Correspondence: J onathan Bae. Address: Division of Hospital Medicine, Department of Medicine, Duke University School of Medicine, Durham, NC 27710, United States. E-mail: jon.bae@duke.edu

Received: September 10, 2012 Accepted: November 8, 2012

Online Published: November 14, 2012

DOI : 10.5430/jha.v2n1p28

URL: http://dx.doi.org/10.5430/jha.v2n1p28

\section{Abstract}

Hospital readmissions present a costly problem for healthcare systems. Engaging care providers in reviewing readmissions may reveal opportunities for reducing readmissions and improving quality. We developed a real-time alerting method that e-mails providers when a discharged patient returns for care (emergency department [ED] or hospital admission) within 30 days. We analyzed the content of alerts to consider frequency of presentation and demographic characteristics of readmitted patients. From 3/15/2011 to 8/31/2011, 1544 alerts (943 ED returns; 601 inpatient readmissions) were generated, representing 621 unique patients (average return time: $12.8 \pm 8.5$ days). Forty-eight faculty received alerts; $88.8 \%$ of alerts were sent to the correct discharging provider. Real-time alerting allows providers to re-engage with readmitted patients and offers a means for evaluating performance. Such systems may also elucidate reasons for readmission by detailing practice patterns and populations at risk for readmission as well as help to design targeted interventions to reduce hospital returns.

\section{Key words}

Patient readmission, Hospital costs, Quality of care, Medical informatics, Patient selection, Cohort identification, Real-time alerts, Quality improvement

\section{Introduction}

Hospitals and healthcare organizations have focused on hospital readmissions as a metric for healthcare quality, an opportunity for improving patient care, and as a target for cost-saving efforts. Among Medicare beneficiaries who were discharged from hospitals, almost $20 \%$ were rehospitalized within 30 days; $34 \%$ were rehospitalized within 60 days ${ }^{[1]}$. The Medicare Payment Advisory Commission (MedPAC) estimates the cost of unplanned rehospitalizations at $\$ 12$ billion per year ${ }^{[1]}$. Although not all rehospitalizations are avoidable, early rehospitalization has been proposed as an important quality-of-care indicator because it may stem from a failure during the preceding hospital stay ${ }^{[2]}$. Accordingly, MedPAC has advised reducing payments to hospitals for readmissions ${ }^{[1]}$, and individual physicians may be held accountable as well. 
Readmissions, however, are driven by multiple factors, and well-defined profiles for identifying patients at risk for preventable rehospitalization are currently lacking. One key factor is the role played by process-of-care issues, which include avoidable circumstances such as incomplete medication reconciliation, failed discharge plans, or complications from prior hospitalization (e.g., deep vein thrombosis; urinary catheter-related infections). MedPAC estimates that approximately $13 \%$ of hospital readmissions are potentially preventable ${ }^{[1]}$, but estimates from other studies range from as low as $5 \%$ to as high as $79 \%{ }^{[3]}$.

Currently, readmission analysis is driven by hospital performance monitoring groups that use administrative and claims data as metrics of hospital performance and reimbursement ${ }^{[4]}$. Such analysis is limited to demographic information, payer status, billing codes, and claims data. However, rehospitalization classification is subjective and time-intensive, and administrative data provide only limited insight into opportunities for improving care delivery and reducing risks of hospitalization. An ideal classification process would include the providers who originally discharged the patient, as they have a unique vantage point from which to judge whether an opportunity for prevention exists.

In this article, we report on our institution's efforts to use open-source tools to configure and deploy a prospective, real-time query of Health Level 7 (HL7) messages ${ }^{[5]}$ that notifies providers when recently discharged patients are readmitted or return to the emergency department (ED), thereby allowing providers the opportunity to review those cases and identify targets for improvement in personal practice, as well as enabling case-by-case analysis of readmissions and ED returns to identify targets for system-wide quality improvement efforts.

\section{Methods}

\subsection{Setting and study population}

Our study took place on the general medicine service of Duke University Hospital (DUH), a 924-bed tertiary-care academic medical center. This service sees approximately 6000 discharges per year, roughly $18 \%$ of which constitute readmissions. The service is staffed by faculty and resident physicians and is divided into five resident and four hospitalist teams. The former include both faculty and resident physicians, while the latter are staffed by faculty alone. These nine teams care for an average of 110 patients daily; approximately 24 physicians ( 15 residents, 9 faculties) are on service at any given time.

Our study involved all patients who presented to DUH (either via ED or admitted to an inpatient service) from 3/15/2011 to $8 / 31 / 2011$ and were discharged from the DUH general medicine service within the prior 30 days. This work was completed as a quality improvement project and reanalyzed for publication after approval by the Duke University Institutional Review Board.

\subsection{Configuration of real-time alerts}

This project used the Duke Integrated Subject Cohort and Enrollment Research Network (DISCERN) application, a real-time cohort recruitment system designed to identify potential participants for enrollment in clinical research. As previously described ${ }^{[5]}$, DISCERN can be configured for any case involving research or quality improvement in which a rules-based, real-time alert is needed but is not available from other health IT systems. For this study, a DISCERN alert was configured to (1) identify patients who were discharged by general medicine providers and then readmitted or seen in the ED within 30 days of discharge and (2) to alert the discharging physician via automated e-mail. DISCERN used data from two sources to create an alert: retrospective data from Duke Medicine's enterprise data warehouse (EDW), and real-time HL7 messages created and exchanged when clinical data systems intercommunicate in support of immediate care (e.g., care actions, orders, patient movement, medical document management, care results, and scheduling). At Duke Medicine, these HL7 messages are exchanged through an integration broker product (Sun eGATE) and routed to the 
appropriate clinical applications. The integration broker handles the processing and translation that enable these applications to exchange information.

Figure 1 describes the alert configuration that combines EDW and HL7 data. A procedure stored in the EDW runs SQL statements every 24 hours and populates a database table containing all discharge information for the last 30 days along with discharging provider data. HL7 messages sent across the integration broker are queried using the open-source MIRTH tool (http://www.mirthcorp.com/ [WebReach]). MIRTH queries the HL7 messages using channels, which are modular programmatic scripts configured by the DISCERN developer to connect to data sources, apply filters, transform data, and re-send the data to another location (either external or another channel).

Figure 1. DISCERN alert process

DISCERN uses the MIRTH engine to listen for HL7 messages exchanged across the integration broker as different health care systems intercommunicate. Channel 1 is configured to listen for all HL7 messages and send those of a certain type to channels devoted to specific DISCERN projects. Channel 2 processes all HL7 ADT (admit, discharge, transfer) messages and subjects them to a series of filters. Filter $1=$ message reflects an ADT event; Filter 2 = ADT type is either A04 (emergency department registration) or A01 (inpatient admission); Filter 3 = location of ED (emergency department) or IP (inpatient). Messages that pass all filters are used to query the enterprise data warehouse (EDW) table that contains all discharges by general medicine providers in the past 30 days. When all conditions are met, the components of the HL7 message (patient ID, location, date, registration time) as well as the information from the EDW table are used to generate

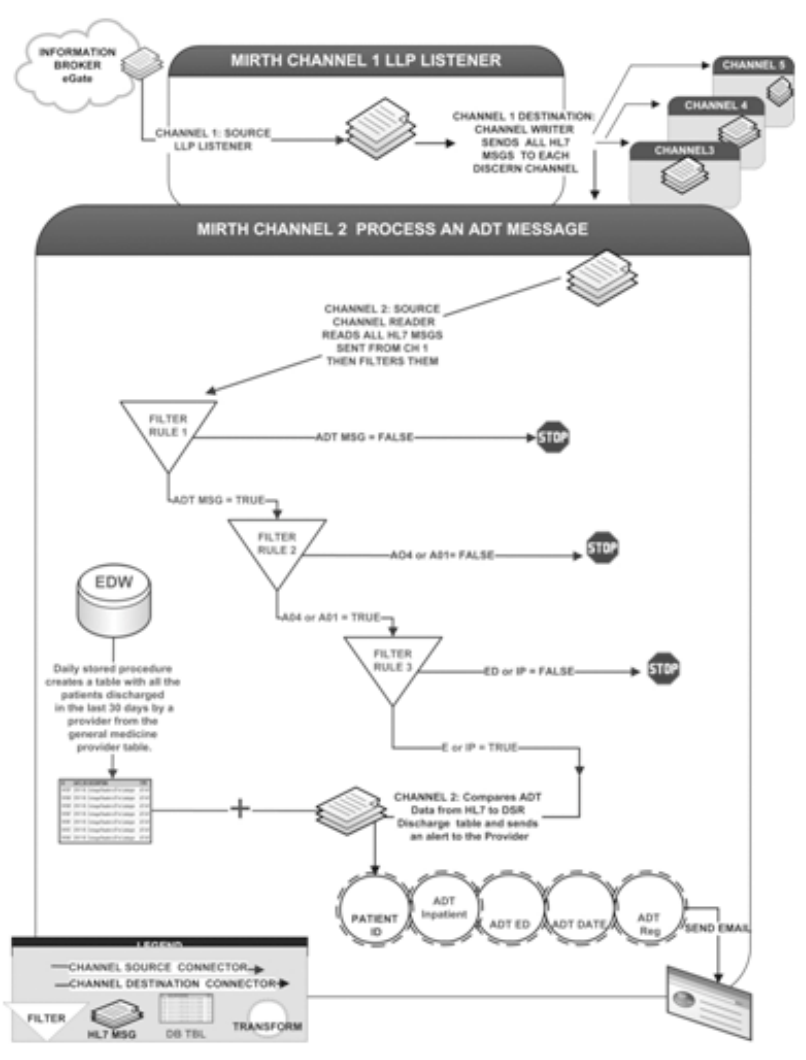
and send an e-mail alert.

Two channels are used in the readmission notification query. Channel 1 is generic feature configured as a Lower Level Protocol (LLP) Listener for all HL7 messages received by the integration broker and feeds them to other channels designed for specific use cases. Channel 2 accepts messages from Channel 1 and filters for any HL7 admission, discharge, transfer messages that indicate a hospital or ED admission. When all filter conditions are met, the patient identifier is removed and used by Channel 2 to query the EDW table that holds discharge information for the previous 30 days. When a match is found, Channel 2 assembles all information into an e-mail template that is sent to the provider associated with the most recent hospitalization and to the project coordinators. The e-mail includes patient name, medical record number, date of discharge, date of ED return or readmission, days since discharge, and discharge and return diagnoses (Figure 2).

\subsection{Alert Review by discharging providers}

Prior to the start of this project, an e-mail was sent to faculty providers on the general medicine service outlining the study's premise. Embedded in the notification itself was a description of the notification's purpose and the possibility that 
the provider might be asked to complete reviews of a readmission. Providers were not expected to interact with the notifications and it was left to them to decide what to do with the information provided. The study team had no capability to track if and when providers interacted with the alerts. Contact information was included in the notification for providers to use if they were incorrectly identified as a discharging physician or if they had questions. Over the course of the project, the alerts were forwarded to 34 resident physicians for their review as well.

Figure 2. DISCERN e-mail notification

An e-mail alert is sent to the discharging provider whenever a patient presents to Duke Medicine ED or inpatient floors within 30 days. Information from the DISCERN query populates the data in the e-mail.



After the notification system had been active for 2 weeks, providers who had received at least one notification were sent an 11-question survey and asked to assess the utility of the e-mail alerts. The survey included questions designed to evaluate how providers were using the alerts, such as "When you received a notification, what did you do with the information?" and "Do you think the notifications have the potential to impact care for your patients (for example: change the way you take care of discharges, etc.)." A second cycle of the surveys were distributed between 4/11/2011 and 6/16/2011 among faculty and resident providers for any readmission that triggered an alert to characterize process-of-care factors, such as discharge practices and coordination of patient care. Questions in this survey included: "Were there any tests/labs pending at the time of discharge?" and "Did the patient have an identified primary care provider?" The survey also asked providers to qualify whether they thought the readmission was preventable, and if so, how this could have been achieved.

\subsection{Data collection and analysis}

In addition to being sent to the discharging physicians, all alerts were stored in the EDW. The project coordinator also received an extract detailing all alerts created during the study period so that they could be evaluated for accuracy of notification distribution. The accuracy of the discharging provider identification was verified by manual chart review.

In addition to basic demographic information of patients with hospital returns, the project team tracked the total number of notifications (in aggregate and by subtype), the number of unique patients, date of discharge, date of readmission or ED return, time since discharge, notification recipient, discharge diagnosis, return diagnosis, and the actual attending physician of record. In order to determine whether there were differences in the number of notifications received by residents versus hospitalist teams, the discharging service and the discharging resident (if the patient was on a resident team) were both tracked. Information was also collected regarding whether the patient was discharged from general medicine or from an alternative service such as pediatrics. Project coordinators used manual chart review to identify service lines. Also, in order to identify populations at high risk for readmission, notifications for patients with sickle-cell disease (which make up a substantial portion of the general medicine readmission totals) as well as alerts for any patient who had $\geqslant 4$ notifications ( "high utilizers" ) during the study period were followed. The study team also reviewed the distribution of discharge diagnoses associated with the index admissions that subsequently generated a notification to determine which diagnosis were most commonly associated with patient return. This was done by review of the billing 
diagnosis associated with the index admission; since there was substantial overlap between multiple billing diagnoses, the study team combined codes into broader groups.

Descriptive statistics were used to calculate percentages of each type of notification (to ED versus inpatient), each type of notification to each service (resident versus hospitalist), ED and inpatient notifications per high-risk group (sickle-cell and high utilizers) and within specified time frames ( $<24$ hours and $<7$ days) as well as the average time until patient return. Descriptive statistics were also used to describe the results of provider survey data.

Figure 3. Weekly notifications of readmissions and ED returns.

The total notifications are plotted by study week as well as the counts of those that are ED returns or inpatient readmissions.

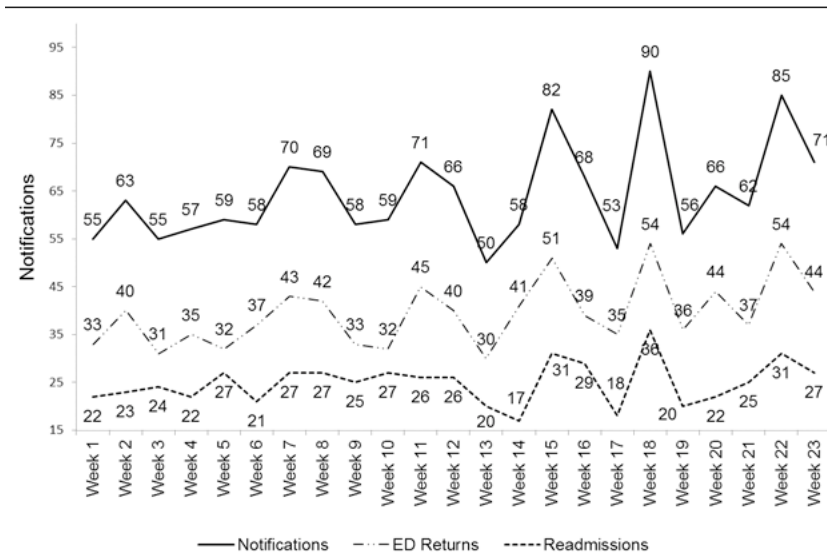

We evaluated the notification system's precision in order to measure its fidelity. Precision would typically be defined as the percentage of all DISCERN alerts with the correctly identified discharging provider, as determined by manual chart review. However, because patients with care episodes that started in the ED and transitioned to an inpatient floor would generate two DISCERN alerts, such a definition would double-count misdirected notifications for the same care episode. We therefore defined precision as the percentage of care episodes with a correctly identified discharging provider detailed in the e-mail alert.

Data were analyzed using Microsoft Excel 2010 (Microsoft; Redmond, WA) and JMP 9.0 (SAS; Cary, NC).

\section{Results}

\subsection{Frequency of alerts}

During the period from 3/15/2011 to 8/31/2011, there were 1544 e-mail notifications representing 621 unique patients sent to 48 faculty physicians who had discharged patients from the general medicine service, an average of $64( \pm 10.4)$ alerts per week (Figure 3). Of these, 943 (61\%) were ED returns and 601 (39\%) were readmissions. A total of 465 ED return alerts were followed by an inpatient admission (ED/IP pair), meaning the discharge provider received both an ED return alert as well as an inpatient admission alert (49.3\% of all ED alerts). Together, the alerts reflect 1079 distinct care episodes: 478 ED-only (44.3\%), $465 \mathrm{ED} / \mathrm{IP}$ pairs (43.1\%), and $136 \mathrm{IP}$-only (12.6\%). The average time until patient return was $12.8 \pm 8.5$ days. Although most notifications (93.8\%) were correctly related to the general medicine discharge service, there was a small proportion discharged from other service lines (Figure 4), indicating less than $100 \%$ precision in alerts.

Of the 1079 total care episodes that returned to DUH, 1013 had been previously discharged from the general medicine service. Of these, $60.6 \%$ (614/1013) originated from resident teams and the remaining 39.4\% (399/1013) originated from hospitalist teams. Resident teams accounted for 59.3\% of ED returns (531/895) and 63.5\% of readmissions (351/553). 
This distribution of patient encounters between resident and hospitalist teams reflects the general distribution of the patient census between the two services (i.e., approximately $2 / 3$ of the patient census are seen by resident teams) and the disparity was not considered clinically significant.

Figure 4. Notification alerts by discharging service line. Most alerts reflected providers discharging patients from general medicine. A small minority of alerts were for patients discharged from other services, reflecting the fact that general medicine providers may round on a different services at times. This provider group includes a proportion of medicine-pediatric faculty who round on both general medicine and pediatrics.

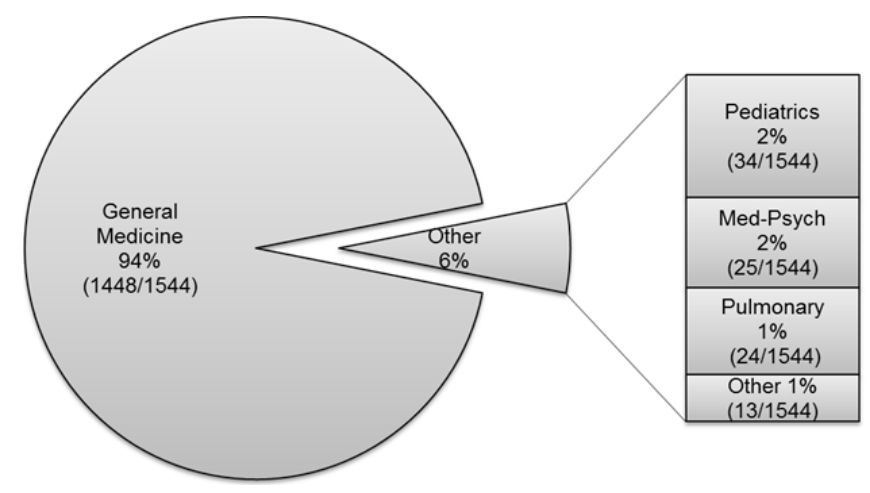

\subsection{Demographics of presenting population}

Table 1 shows the demographic characteristics of the readmitted population as identified by DISCERN. Patients were predominantly female and equally black or white by racial group. Patients of Hispanic ethnicity and/or racial groups other than white or black were underrepresented by population percentage. The average length of stay on the index admission was 5.20 days (SD 4.38).

Table 1. Demographics of returned general medicine patients

\begin{tabular}{lll}
\hline Demographic category & N & Percent \\
\hline Females & 343 & $55.2 \%$ \\
Race & & \\
American Indian & 4 & $0.6 \%$ \\
Asian & 4 & $0.6 \%$ \\
Black & 310 & $49.9 \%$ \\
White & 284 & $45.7 \%$ \\
Other & 19 & $3.1 \%$ \\
Hispanic ethnicity & 13 & $2.1 \%$ \\
Average Age & 56.5 years \\
\hline
\end{tabular}

Given the high costs associated with readmissions, as well as the implications for patient safety and quality of care, the readmitted population was scrutinized in order to elucidate the factors that contributed to the majority of readmission events. Of the 601 distinct care episodes that involved a readmission and generated an alert, 553 involved a patient who was discharged by a general medicine provider during the index admission. A total of 521 of these alerts (94.2\%) could be 
classified as belonging to special subpopulations (classifications were not mutually exclusive). High utilizers generated $42.9 \%$ of these readmission alerts (Figure 5), and 33.3\% of readmission alerts occurred within 7 days of the index admission; $6.1 \%$ of these occurred within 24 hours of discharge. Many high utilizers were also sickle-cell patients, who accounted for $11.9 \%$ of all alerts.

Figure 5. General Medicine Populations Presenting to Duke University Hospital.

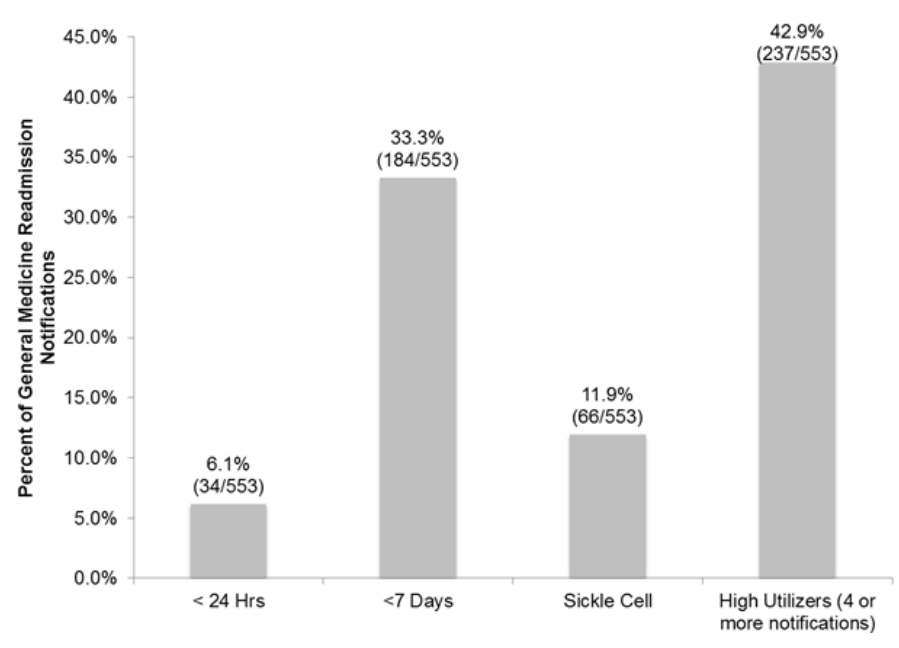

In review of the index admission diagnoses, there were 471 (of 1538) encounters for which data was not available. Of the remaining 1067, 69.1\% (737/1067) came from 25 discharge diagnosis groups. The remaining 30.9\% (330/1067) index admission diagnoses came from 130 different diagnosis groups (results shown in Figure 6). This must be interpreted with some caution as primary diagnosis group does not capture the host of co-morbidities which also may have driven the initial hospitalization and reflect the patient's overall health.

Figure 6. Distribution of discharge diagnoses from index admission.

Discharge diagnosis was grouped due to overlap. There were 471 encounters that did not have discharge information available. For the remaining 1067, 737 (69.1\%) came from 25 discharge diagnosis groups. The remaining 330 (30.9\%) came from 130 additional discharge diagnoses.

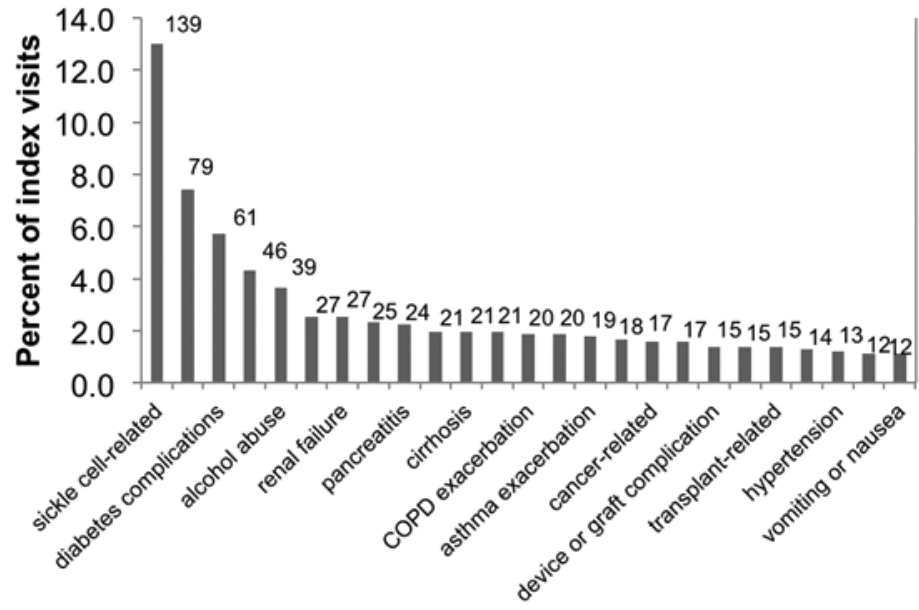

\subsection{Evaluation of alerting fidelity}

Precision was calculated to define the degree of inaccuracy of EDW information in identifying the discharging provider. Precision was defined as the percentages of all DISCERN alerts with the correctly identified discharging provider and 
service line, as determined by manual chart review. At the time an alert was created, the EDW provided the accurate discharging provider in $88.8 \%$ (958/1079) of all care episodes. In comparing the discharging provider of record from the

Table 2. Survey results for alert utility assessment

\begin{tabular}{|c|c|c|c|c|}
\hline Results category & $\mathbf{n}$ & $\%$ & Total N & Missing \\
\hline \multicolumn{5}{|l|}{ Patient characteristic associated with readmission } \\
\hline$>3$ hospital admissions within recent 12 months & 67 & 50.4 & 114 & 19 \\
\hline$>10$ ED admissions within recent 12 months & 32 & 28.8 & 111 & 22 \\
\hline Takes $\geq 6$ medications & 106 & 84.8 & 125 & 8 \\
\hline Psychiatric diagnosis & 24 & 19.2 & 125 & 8 \\
\hline Diabetes & 35 & 35.4 & 99 & 34 \\
\hline Substance abuse & 18 & 14.4 & 125 & 8 \\
\hline Chronic obstructive pulmonary disease & 9 & 9.1 & 99 & 34 \\
\hline Chronic heart failure & 14 & 11.2 & 125 & 8 \\
\hline \multicolumn{5}{|l|}{ Discharge documentation practices } \\
\hline Medications on discharge matched medications on summary & 119 & 96.0 & 124 & 9 \\
\hline Discharge instructions had medication with doses & 123 & 99.2 & 124 & 9 \\
\hline Discharge instructions had medications with route of administration & 123 & 99.2 & 124 & 9 \\
\hline Discharge instructions had medications continued, discontinued, changed, or initiated & 122 & 100 & 122 & 11 \\
\hline Discharge instructions had medication instructions in plain language & 111 & 89.5 & 124 & 9 \\
\hline Pending labs or test at time of discharge (with clear follow-up plan) & 20 & 16.1 & 124 & 9 \\
\hline Discharge forms identified provider ( $\&$ contact information) to call for questions/concerns & 95 & 77.9 & 122 & 11 \\
\hline Discharge summary/instructions included patient functional status & 66 & 53.7 & 123 & 10 \\
\hline \multicolumn{5}{|l|}{ Post-discharge care } \\
\hline Discharge summary faxed to an outpatient provider & 109 & 94.8 & 115 & 18 \\
\hline Patient had access to medications or filled prescription upon discharge & 92 & 74.2 & 124 & 9 \\
\hline Cannot determine & 32 & 35.8 & - & - \\
\hline Patient had an identified PCP & 79 & 83.2 & 95 & 38 \\
\hline \multicolumn{5}{|l|}{ Follow-up appts with outpatient provider to occur within: } \\
\hline 1-7 days & 69 & 57.5 & 83 & 13 \\
\hline 8-14 days & 21 & 17.5 & 104 & \\
\hline$>14$ days & 16 & 13.3 & 120 & \\
\hline Patient made follow-up appt or had clinic visit prior to readmission & 51 & 44.7 & 114 & 19 \\
\hline Cannot determine & 23 & 20.2 & - & - \\
\hline $\begin{array}{l}\text { Social work, physical/occupational therapy, or PRM during preceding hospitalization that identified } \\
\text { barriers to care }\end{array}$ & 49 & 39.8 & 123 & 10 \\
\hline Patient discharged with home health, personal care services, home PT/OT, or other & 23 & 25.6 & 90 & 43 \\
\hline Documented concerns of patient adherence to prescribed therapies & 32 & 36.4 & 88 & 45 \\
\hline Patient known to have access to reliable transportation & 61 & 71.8 & 85 & 48 \\
\hline Transportation status unknown & 15 & 15.7 & - & - \\
\hline
\end{tabular}

appt, appointment; ED, emergency department; OT, occupational therapy; PCP, primary care provider; PT, physical therapy 
alerts to those listed in the EDW in December 2011 (at least 3 months after the original alert), 31.3\% (31/99) of these index admissions were later associated with the correct discharge provider.

\subsection{Alert utility provider surveys}

A survey to assess the content of the notifications and their utility was distributed early in the project. The survey was distributed to 23 faculty providers, 10 of whom responded (43.5\%). When asked whether the received notifications had the potential to affect patient care, 3 providers indicated that it had a "major impact"; 5 indicated the potential impact was "slight." Upon receiving the notifications, providers stated their most common response was to review the patient chart $(n=7)$, discuss the case with the medical team $(n=4)$, visit the patient in the ED or hospital $(n=2)$, or contact the ED or admitting physician $(n=1)$. However, it must be noted that physician's compliance with this survey may also be correlated with an increased likelihood to interact with the notification itself and a desire to learn from the readmission episode.

A survey to assess process-of-care factors was sent to providers receiving alerts near the end of the project. This survey was distributed to 31 attending providers and 24 resident providers for 218 readmission alerts. Responses were received for 146 readmission cases; 2 surveys were excluded due to missing data. Among the cases included for analysis, 133 (92.4\%) were an unscheduled readmission with $20 \%$ considered preventable and $7 \%$ possibly preventable. Among these cases, 93 cases (69.9\%) were readmitted due to complications related to the index admission. Cases were found to display typical associated health risk factors ${ }^{[6]}$ for readmission (results shown in Table 2).

\section{Discussion}

Our study examined the use of a real-time notification system to alert discharging physicians of their patient's return to the emergency department or readmission to the hospital. Over a 3-month period, the system identified 621 patients and engaged 82 providers (attending physicians and residents). A major advantage of this type of real-time notification system is its generalizability to other healthcare systems. DISCERN itself is a code written to solve the limitations of the current information technology at our institution; specifically, the weak capacity for communicating data at care transitions or across encounters. However, the overall scheme for detecting HL7 messages to identify patient readmissions can be adopted by any organization. As healthcare systems continue to adopt electronic medical records and information systems, a better-integrated system could resolve many of the issues related to real-time identification, including accuracy and precision, and could aide in the provision of seamless care delivery across therapeutic settings.

Real-time alerting systems are common in healthcare settings. Computerized notification systems have been used to alert physicians to critical laboratory values ${ }^{[7]}$, identify possible adverse drug events ${ }^{[8,9]}$, provide surveillance for hospital-acquired infections and antibiotic use ${ }^{[10]}$, and to identify and recruit patients to clinical trials ${ }^{[5]}$. The use of provider alerts has been shown to change physician behavior and reduce the frequency of adverse events ${ }^{[7,9,10,11]}$.

Computerized alert systems have also been used to identify hospitalizations, primarily as a means of alerting primary care physicians and for the purpose of coordinating care ${ }^{[12]}$. However, to the best of our knowledge, the use of real-time notifications to identify readmissions for discharging providers has not been described in the literature and may represent a novel application of this technology, although the published literature contains a number of descriptions of models designed to alert providers of patients at risk for readmission in real time ${ }^{[13,14,15]}$.

By focusing on timing or disease-specific groups, we may be able to identify populations at high risk of readmission for potential intervention. This may include strategies such as the development of a sickle-cell day hospital ${ }^{[16,17]}$ or intensive care transitions interventions ${ }^{[18]}$, which have been shown to reduce healthcare utilization for certain groups. One health system succeeded in reducing their readmission rate by $6.4 \%$ through implementation of a nurse navigator case management workflow, in which registry-based reports are used to identify high-risk cases and proactively manage their care ${ }^{[19]}$. In addition, there is evidence to suggest that readmissions occurring closer to the time of discharge are more likely 
to reflect a process-of-care issue or failure to schedule timely follow-up appointments ${ }^{[20]}$, as opposed to those that occur later and are more likely to reflect progression of a chronic disease. Identifying readmissions that occur within a particular interval (24 hours; 7 days) for root-cause analysis or a peer-review forum may also reveal opportunities to improve care delivery.

Our study faced a number of challenges. First, there is an inherent lack of precision in identifying the correct discharging attending, due in part to inaccuracies when data are first placed in the EDW. However, this effect was relatively small, as $88.8 \%$ of total encounters had correctly identified the provider of record. Over time, as additional data sources (e.g., billing data) are integrated and all encounter-related records are closed, these inaccuracies are corrected, as reflected by the fact that $33.3 \%$ notifications that were originally associated with wrong providers were later found to be correct in the EDW, more than 3 months later.

We also inadvertently captured a small number of returns discharged from service lines other than general medicine. This error arose from the fact that patients discharged from general medicine were identified based on a list of providers who round on general medicine units; because a minority of providers round on multiple services, such as pediatrics or pulmonary in addition to general medicine, occasional alerts for readmission would occur for patients on a different service. This highlights a key limitation of real-time alerts in health care: inaccuracies in identifying events and alerting the appropriate individuals can delay the transfer of critical information needed for patient care. In the present application, the identification of alerts in real time creates an opportunity for providers to intervene in care- for example, to visit a patient in the ED and collaborate with physicians to coordinate care - but this was not part of the standard of care. Thus, data inaccuracies were thought to be more allowable, because the risk is only that of a misdirected alert and distribution of protected health information.

At present, we are not able to evaluate the sensitivity and specificity of the real-time alert. To do so, we would need to be able to identify not only false negatives - i.e., real "hits" missed by the system, but also true negatives - the number of all potential hits "passed on" by DISCERN. Identifying the latter would require us to collect all HL7 messages and store them for later parsing by a scripting language, and we currently lack the hardware needed to store this large volume of information.

An additional challenge we experienced was "alert fatigue." An increasing number of alerts may result in loss of effective information conveyance ${ }^{[13,21]}$, creating a barrier to the effective use of notification systems. Alert fatigue has been described in the context of patient work flows; specifically, on-screen alerts about critical laboratory values or potential drug interactions within computerized physician order entry (CPOE) systems ${ }^{[13]}$. Because our notification system used e-mail, interruptions of provider workflow may have been limited; however, similar difficulties arising from e-mail alert fatigue can be imagined as the volume of notifications increases. On average, a physician on service (or recently on service) received three to four e-mail notifications each week. In this context, the imprecision related to notification identification becomes paramount, as provider confidence in notifications is directly proportional to their accuracy. Additionally, if the imprecision is high, the risk that providers will ignore the alert increases - a limitation particular to e-mail notifications (versus text page or on-screen alerts), which require the intended recipients to check and read their e-mail. Unfortunately, the project team had no way to track whether providers were interacting with the alerts once they received them.

We also encountered data management issues that deserve discussion. In its present form, our EDW does not allow us to identify and subsequently notify resident discharging physicians associated with readmissions for the purpose of providing performance feedback or to engage them in provider review. Instead, doing so required time-intensive manual chart review. This shortcoming arose from the limitations of the current electronic medical record, which identifies only faculty physicians currently associated with patients, highlighting the importance of anticipating data needs before constructing an alert system. 
A major limitation of this study is the lack of quantitative data regarding how discharging providers used the alerts to follow-up with patients during the readmission episode. Providers were given no instruction for follow-up once a notification was received; however, anecdotal reports suggest that providers visited patients who had returned and in some cases helped coordinate care for eventual discharge. As noted above, providers were surveyed early in the process (at 2 weeks) and solicited for feedback on the notifications themselves. Although the sample size of only 10 respondents was admittedly small, this does suggest that these notifications have the potential to affect care delivery. Further evaluation of provider interaction with these alerts, and a detailed exploration of actions prompted by receiving them, represents an important area for future study.

\section{Conclusion}

Real-time notification systems have multiple applications in terms of addressing the problem of patient readmissions and are adaptable to a diverse array of health care settings. In particular, they offer a possible mechanism for providing performance feedback to discharging providers, allowing them to understand the outcomes of their care practice and identify targets for improvement. Such performance feedback and provider review of readmissions could potentially set the stage for additional quality improvement interventions through the identification of process improvement targets. Future directions could include focusing on disease-specific readmissions or high-risk groups for targeted intervention as well as further study of the impact of the notifications on provider practice. However, additional research is needed in order to better describe the effect such notices have on provider practice, and to elucidate optimal strategies for implementing such alerts and integrating them into clinical workflows.

\section{Acknowledgements}

We thank the following faculty from the Duke Department of Medicine for all of their support, advice, and mentorship: Diana McNeill, MD; G. Ralph Corey, MD; David Gallagher, MD; Ben Powers, MD; Brian Griffith, MD; Thomas LeBlanc, MD; Gary Cox, MD; Aimee Zaas, MD; Mary Klotman, MD; Harvey Cohen, MD; and Suzanne Woods, MD. We also thank Duke University Hospital Medicine and all general medicine faculty and residents for supporting and participating in this project. Finally, we thank Howard Shang, Director of Information Management, Duke Health Technology Services, for supporting this project; and Jonathan McCall, MS, from the Duke Clinical Research Institute, Durham, NC, for editorial assistance in preparing this paper.

\section{References}

[1] Jencks, S., \& Williams, M. Rehospitalizations among patients in the Medicare fee-for-service program. New England Journal of Medicine. 2009; 360 (14): 1418-1428. PMid: 19339721 http://dx.doi.org/10.1056/NEJMsa080356

[2] Goldfield, N. I., McCullough, E. C., Hughes, J. S., Tang, A. M., Eastman, B., Rawlins, L. K., \& Averill, R. F. Identifying potentially preventable readmissions. Health Care Financing Review. 2008; 30 (1): 75-91. PMid:19040175

[3] Van Walraven, C., Bennett, C., Jennings, A., Austin, P. C., \& Forster, A. J. Proportion of hospital readmissions deemed avoidable: a systematic review. Canadian Medical Association Journal. 2011; 183 (7): E391-E402. http://dx.doi.org/10.1503/cmaj.101860

[4] Centers for Medicare and Medicaid Services website. Outcome measures [Internet]. Available from: http://www.cms.gov/Medicare/Quality-Initiatives-Patient-Assessment-Instruments/HospitalQualityInits/OutcomeMeasures.html. Accessed June 28, 2012.

[5] Ferranti, J., Gilbert, W., McCall, J., Shang, H., Barros, T., \& Horvath, M. M. The design and implementation of an open-source, data-driven cohort recruitment system: the Duke Integrated Subject Cohort and Enrollment Research Network (DISCERN). Journal of the American Medical Informatics Association. 2011; 19 (e1): 368-e75.

[6] Metzger, J. \& Lorincz, C. Preventing hospital readmissions: the first test for continuity of care. Computer Science Consortium [Internet]. Available from: http://assets1.csc.com/health_services/downloads/CSC_Preventing_Hospital_Readmission.pdf. Accessed September 7, 2012. 
[7] Rind, D., Safran, C., Phillips, R. S., Wang, Q., Calkins, D. R., Delbanco, T. L., Bleich, H. L., \& Slack, W. V. Effect of computer-based alerts on the treatment and outcomes of hospitalized patients. Archives of Internal Medicine. $1994 ; 154$ (13): 1511-17. http://dx.doi.org/10.1001/archinte.1994.00420130107014

[8] Raschke, R., Gollihare, B., Wunderlich, T. A., Guidry, J. R., Leibowitz, A. I., Peirce, J. C., Lemelson, L., Heisler, M. A., \& Susong, C. A computer alert system to prevent injury from adverse drug events: development and evaluation in a community teaching hospital. Journal of the American Medical Association. 1998; 280 (15): 1317-1320. http://dx.doi.org/10.1001/jama.280.15.1317

[9] Kuperman, G., Teich, J. M., Tanasijevic, M. J., Ma'Luf, N., Rittenberg, E., Jha, A., Fiskio, J., Winkelman, J., \& Bates, D. W. Improving response to critical laboratory results with automation: results of a randomized controlled trial. Journal of the American Medical Informatics Association. 1999; 6 (6): 512e22. http://dx.doi.org/10.1136/jamia.1999.0060512

[10] Evans, R. S., Larsen, R. A., Burke, J. P., Gardner, R. M., Meier, F. A., Jacobson, J. A., Conti, M. T., Jacobson, J. T., \& Hulse, R. K. (Computer surveillance of hospital-acquired infections and antibiotic use. Journal of the American Medical Association. 1986; 256 (8): 1007-1011. http://dx.doi.org/10.1001/jama.1986.03380080053027

[11] Bates, D., \& Gawande, A. Improving safety with information technology. New England Journal of Medicine. 2003; 348 (25): 2526-2534. http://dx.doi.org/10.1056/NEJMsa020847

[12] Moran, W. Where are my patients? It is time to automate notification of hospital use to primary care practices. Southern Medical Journal. 2012; 105 (1): 18-23. http://dx.doi.org/10.1097/SMJ.0b013e31823d22a8

[13] Amarasingham, R., Moore, B. J., Tabak, Y. P., Drazner, M. H., Clark, C. A., Zhang, S., Reed, W. G., Swanson, T. S., Ma, Y., \& Halm, E. A. An automated model to identify heart failure patients at risk for 30-day readmission or death using electronic medical record data. Medical Care. 2010; 48 (11): 981-988. http://dx.doi.org/10.1097/MLR.0b013e3181ef60d9

[14] Billings, J., Dixon, J., Mijanovich, T., \& Wennberg, D. Case finding for patients at risk of readmission to hospital: development of algorithm to identify high-risk patients. BMJ. 2006; 333: 327 (abstract). http://dx.doi.org/10.1136/bmj.38870.657917.AE

[15] Kansagara, D., Englander, H., Salanitro, A., Kagen, D., Theobald, C., Freeman, M., \& Kripalani, S. Risk prediction models for hospital readmission: a systematic review. Journal of the American Medical Association. 2011; 306 (15): 1688-1698. http://dx.doi.org/10.1001/jama.2011.1515

[16] Benjamin, L. J., Swinson, G. I., \& Nagel, R. L. Sickle cell anemia day hospital: an approach for the management of uncomplicated painful crises. Blood. 2000; 95 (4): 1130-1136. PMid:10666181

[17] Adewoye, A. H., Nolan, V., McMahon, L., Ma, Q., \& Steinberg, M. H. Effectiveness of a dedicated day hospital for management of acute sickle cell pain. Haematologica. 2007; 92 (6): 854-855. http://dx.doi.org/10.3324/haematol.10757

[18] Coleman, E. A., Parry, C., Chalmers, S., \& Min, S. J. The care transitions intervention: results of a randomized controlled trial. Archives of Internal Medicine. 2006; 166 (17): 1822-8. http://dx.doi.org/10.1001/archinte.166.17.1822

[19] Comission for Care Management Certification. Communicate, engage, transform: Bon Secours case managers part of a robust team enhancing health care delivery and patient health. CCMC Issue Brief [Internet]. 2011; 2 (5). Available from:

http://ccmcertification.org/sites/default/files/downloads/briefs/. Accessed September 7, 2012.

[20] Hernandez, A. F., Greiner, M. A., Fonarow, G. C., Hammill, B. G., Heidenreich, P. A., Yancy, C. W., Peterson, E. D., \& Curtis, L. H. Relationship between early physician follow-up and 30-day readmission among Medicare beneficiaries hospitalized for heart failure. Journal of the American Medical Association. 2010; 303 (17): 1716-1722. http://dx.doi.org/10.1001/jama.2010.533

[21] Shah, N., Seger, A. C., Seger, D. L., Fiskio, J. M., Kuperman, G. J., Blumenfeld, B., Recklet, E. G., Bates, D. W., \& Gandhi, T. K. Improving acceptance of computerized prescribing alerts in ambulatory care. Journal of the American Medical Informations Association. 2006; 13 (1): 5-11. http://dx.doi.org/10.1197/jamia.M1868 